\title{
ELASTIC ANALYSIS OF ADHESION STRESSES FOR THE DESIGN OF A STRENGTHENING PLATE BONDED TO A BEAM
}

\author{
Tim Stratford, ${ }^{a^{*}}$ John Cadei ${ }^{b}$ \\ ${ }^{a}$ Institute for Infrastructure and Environment, The University of Edinburgh, UK \\ ${ }^{\mathrm{b}}$ Independent Consultant, Guildford, UK \\ * Corresponding author. AGB Building, The King's Buildings, Edinburgh, EH9 3JL, UK. \\ Tel: +44 131 6505722; Fax: +44 131 6506781. E-mail: Tim.Stratford@ed.ac.uk
}

\begin{abstract}
This paper presents methods for determining the elastic shear and peel stresses in an adhesive joint between a strengthening plate and a beam. Both closed-form and finite-difference solutions are given, allowing loading, temperature effects and plate prestrain to be considered in design. The method can be used to design strengthened beams with section properties that change along the beam to be examined (such as tapered plates), and can also be used to determine the sensitivity of an adhesive joint to bond defects. The results of some typical load cases and geometries are presented to illustrate the significance of adhesive stresses.
\end{abstract}

\section{KEYWORDS}

Adhesive joint, Stress analysis, Plate bonding, Cast iron, FRP composite, Design, Defects

\section{INTRODUCTION}

Strengthening beams and columns by bonding plates to their surfaces is an effective method for extending the life of ageing infrastructure. Plate bonding relies critically on the strength of the adhesive joint, which must be designed to have adequate strength. However, the design of adhesive joints is rarely encountered in construction.

Plate bonding initially developed using steel plates, but has gained new impetus with the use of lightweight fibre-reinforced-polymer (FRP) composite materials. Both tests and theoretical work have shown that the strength of the adhesive joint is governed by stress concentrations which occur due to the mismatch of strains in the beam and plate across the adhesive layer. Concentrations of adhesive stress occur in the vicinity of geometric discontinuities, such as those shown in figure 1. Geometric 
discontinuities include bond defects in the adhesive layer, steps in the number of strengthening plates, or (in particular) at the end of a strengthening.

This paper describes a method for designing the adhesive joint between a strengthening plate and a beam. The analysis is general, although it was originally developed for FRP strengthening applied to metallic structures [1]. The method predicts stress distributions along the adhesive joint and can be used to failure of the adhesive, or the substrates in the immediate vicinity of the joint, failure modes typically observed in adhesive joints involving metallic or FRP substrates. Failure of adhesive joints with weaker substrates (such as concrete or timber) usually occurs at some distance from the joint (for example, along the flexural reinforcement), a failure mode that is not considered in this paper.

\section{METHODS OF ANALYSING ADHESIVE JOINTS}

Bonded joints have been used since the 1930s, but it is only relatively recently that this technology has been transferred to the construction industry. Adhesive joints in construction are often on a larger scale than those in the automotive or aerospace industries (for example), and behave in different ways. Furthermore, construction projects are one-offs and it is not economic to base design on test results, unlike other industries with long production runs. Consequently, it is important to have realistic models for the adhesive joint strength.

Two approaches can be used to predict the failure of adhesive joints: a stress analysis, or a fracture mechanics approach. Fracture mechanics examines the energy required for unstable crack propagation along the joint; however, this approach has yet to be successfully applied to infrastructure strengthening applications [2].

After the adhesive has cured, the strengthening plate and beam act compositely, with load transferred between them by a combination of shear stresses (parallel to the joint) and peel stresses (normal to the joint). A stress analysis can be used to predict the distributions of shear and peel stress along the strengthened beam, for comparison to the limiting strength of the adhesive joint.

Several closed-form stress analyses are available that predict the distribution of bond stresses along a plate bonded to a beam, for example, see references [3] to [10]. These all assume that the adhesive is linear-elastic, but involve a variety of simplifying assumptions. High-order solutions (such as the analysis presented by Yang, Teng and Chen [9]) model the variation in stress through the thickness of the adhesive layer, but are complex. Lower-order solutions (such as the method presented 
by Deng, Lee and Moy [10]) are simpler, although they assume that the stress does not vary through the adhesive thickness and hence do not satisfy the requirement that the adhesive stress at the free end of the adhesive is stress free.

The motivation behind the approach presented in this paper was the lack of guidance for designing FRP strengthening bonded to metallic structures. The method was partially included in a recently published report 'Strengthening metallic structures using externally-bonded FRP' [1], prior to which the maximum adhesive stress had commonly been estimated by applying a factor of five to the shear stress obtained from a plane sections analysis [11]. An analysis was required that is easily accessible in the design office, hence a low order, linear-elastic, bond analysis was selected for the ease with which results can be obtained and visualised. In recognition of the assumptions made, a consistent low-order analysis is suggested for determining the limiting adhesive stresses from coupon tests.

\section{THE BOND STRESS ANALYSIS}

The bond analysis allows the distribution of shear stress $\left(\tau_{a}\right)$ and peel stress $\left(\sigma_{a}\right)$ along an adhesive joint to be determined. The following sections describe the bond analysis conceptually and give examples of its use. The detailed bond analysis is derived in Appendix A, which is valid for strengthened beams with a constant cross-section.

\subsection{Definitions and assumptions}

Figure 2 summarises the geometric and material properties of the strengthened beam: $E \& G$ are the Young's modulus and shear modulus, $A$ is the cross-sectional area, $I$ is the second moment of area, and $\alpha$ is the coefficient of thermal expansion. The subscripts ' $b$ ', ' $p$ ', and ' $a$ ' refer to the beam, plate and adhesive respectively. The position along the beam is defined by the coordinate ' $x$ ', measured from the geometric discontinuity, and the vertical position is defined by $y$.

The shear stress analysis assumes that the curvatures in the beam and plate are equal (since this allows the shear stress and peel stress equations to be uncoupled). This assumption is not made in the peel stress solution. 


\subsection{Lack-of-fit across the adhesive joint}

The stresses in the adhesive joint are due to the mismatch in strains between the bonded surfaces of the beam and the plate, resulting from load applied after cure of the adhesive joint. The lack-of-fit strains can be found by considering the deformation of the beam and plate if there was no adhesive joint. For example, figure 3 shows a strengthened beam carrying pure moment. If there was no adhesive joint, no load would be applied to the plate and the change in plate strain $\left(\Delta \varepsilon_{p a}\right)$ and curvature $\left(\Delta \psi_{p}\right)$ would be zero. The strain at the bonded surface of the beam $\left(\Delta \varepsilon_{b a}\right)$ and the beam curvature $\left(\Delta \psi_{b}\right)$ are non zero, and can found using engineering beam theory.

If the same moment is applied to a beam with bonded strengthening, the lack-of-fit strain across the adhesive joint is $\Delta \varepsilon_{p b}=\Delta \varepsilon_{p a}-\Delta \varepsilon_{b a}$ (eqn. A5), and the lack-of-fit curvature is $\Delta \psi_{p b}=\Delta \psi_{p}-\Delta \psi_{b}$ (eqn. A6). The bond analysis in Appendix A predicts the distributions of adhesive shear $\left(\tau_{a}\right)$ and peel $\left(\sigma_{a}\right)$ stress due to the lack-of-fit strain $\left(\Delta \varepsilon_{p b}\right)$ and curvature $\left(\Delta \psi_{p b}\right)$.

\section{BOND STRESSES IN TYPICAL SIMPLE LOAD CASES}

A number of typical load cases are investigated below to illustrate the use of the closed-form bond analysis. A cast iron beam, strengthened with CFRPis analysed, using the section properties given in figure 4. The beam carries a brick jack arch bridge deck and requires strengthening due to increased traffic loads. The dead loads present at the time of strengthening do not result in stresses in the adhesive joint. (For ease of reference, this is similar to the case considered in reference [1], appendix A9).

\subsection{Load case 1. Applied load}

After forming the adhesive joint, the strengthened beam is required to carry an additional distributed load of $40 \mathrm{kN} / \mathrm{m}$, which is a typical live load for a cast iron bridge beam to carry due to modern traffic. (This load is $10 \%$ higher than a completed bridge strengthening project).

The resultant change in bending moment is $\Delta M_{b}=20 x^{2}-80 x-100 \mathrm{kNm}$, where $x$ is measured from the end of the plate (as shown in figure 4). The moment at the end of the plate is $\Delta M_{b}=-100 \mathrm{kNm}$. (It is often be sufficient to neglect the variation of $\Delta M_{b}$ with $x$, and use a constant value equal to the moment at the end of the plate). 
Following the approach shown in figure 3, the strains and curvatures in the beam and plate (in the absence of the adhesive joint) are given by (eqns. A3 \& A4, Appendix A):

$$
\begin{array}{ll}
\Delta \varepsilon_{b a}=\left(-0.176 x^{2}+0.71 x+0.88\right) \times 10^{-4} & \Delta \varepsilon_{p a}=0 \\
\Delta \psi_{b}=\left(0.69 x^{2}-2.77 x-3.46\right) \times 10^{-4} / \mathrm{m} & \Delta \psi_{p}=0
\end{array}
$$

The resulting lack-of-fit across the adhesive joint is thus (eqn. A5):

$$
\begin{aligned}
& \Delta \varepsilon_{p b}=\left(0.176 x^{2}-0.71 x-0.88\right) \times 10^{-4} \\
& \Delta \psi_{b}=\left(-0.69 x^{2}+2.77 x+3.46\right) \times 10^{-4} / \mathrm{m}
\end{aligned}
$$

The adhesive shear and peel stresses are plotted in figure 5, which shows a maximum shear stress of 4.2 MPa and a maximum tensile peel stress of 2.5 MPa. The zone of influence of the shear stress $(200 \mathrm{~mm})$ is longer than that of the peel stress $(75 \mathrm{~mm})$.

These maximum stresses can be reduced by curtailing the strengthening plates in regions of low bending stress. For example, the end of the strengthening plate could be extended to $500 \mathrm{~mm}$ from the support, reducing the bending moment at the end of the plate to $56 \mathrm{kNm}$ and the peak shear stress due to the applied load to $2.4 \mathrm{MPa}$ (a $45 \%$ reduction).

\subsection{Load case 2. Uniform temperature rise of $30^{\circ} \mathrm{C}$}

The coefficients of thermal expansion of a metal beam and a carbon FRP strengthening plate can be very different and consequently it is very important to consider the thermal mismatch stresses due to differential thermal expansion.

A uniform temperature rise of $\Delta T=30^{\circ} \mathrm{C}$ is considered for load case 2 . This represents a strengthening plate applied at a temperature of $15^{\circ} \mathrm{C}$, the temperature of which rises to $45^{\circ} \mathrm{C}$ during the summer. (The temperature of a beam on the exposed face of a bridge deck might easily rise to $45^{\circ} \mathrm{C}$; for example, see the UK Highways Agency design requirements [12]).

The lack-of-fit due to a temperature rise of $\Delta T=30^{\circ} \mathrm{C}$ after forming the adhesive joint is described by (eqns. A3, A4 \& A5):

$$
\begin{array}{lll}
\Delta \varepsilon_{p a}=30 \times 10^{-6} & \Delta \varepsilon_{b a}=330 \times 10^{-6} & \Delta \varepsilon_{p b}=-300 \times 10^{-6} \\
\Delta \psi_{p}=0 & \Delta \psi_{b}=0 & \Delta \psi_{p b}=0
\end{array}
$$


The adhesive stress distributions are shown in figure 5. The maximum shear stress is $13.8 \mathrm{MPa}$, and the maximum peel stress of 7.9 MPa. Note that the peak adhesive stresses due to the temperature increase are three times larger than those due to the applied load (case 1).

\subsection{Load case 3. Combined applied load and temperature rise}

The adhesive stress distributions due to the combination of applied load and temperature rise are also shown in figure 5. The maximum stresses due to applied load and temperature are $18 \mathrm{MPa}$ and $10 \mathrm{MPa}$.

\subsection{Load case 4. The situation after the release of a prestressed plate}

A strengthening plate can be prestressed using a jacking system. The jacking force is transferred to the adhesive layer after the adhesive has set. If the pre-strain in the plate prior to forming the adhesive joint is $0.05 \%$, the lack-of-fit strains are:

$$
\begin{array}{lll}
\Delta \varepsilon_{p a}=-0.5 \times 10^{-3} & \Delta \varepsilon_{b a}=0 & \Delta \varepsilon_{p b}=-0.5 \times 10^{-3} \\
\Delta \psi_{p}=0 & \Delta \psi_{b}=0 & \Delta \psi_{p b}=0
\end{array}
$$

If an auxiliary end anchorage device is not provided, prestress results in high adhesive stresses at the end of the plate, as shown in figure 5. The peak shear stress is $23 \mathrm{MPa}$ and the peak peel stress is $13 \mathrm{MPa}$, both of which are higher than any of the previous load cases.

\subsection{Load case 5. A clamping force applied at the end of the plate.}

In this case, a clamping force of $10 \mathrm{kN}$ is applied at the end of the plate (acting so as to push the end of the plate towards the beam). This load case can be used to assess the beneficial effect of clamping the end of a bonded plate to resist live load peel stresses. A $10 \mathrm{kN}$ clamping force could easily be applied using a mechanical anchorage, with bolts tightening a clamp that traps the FRP against the bottom flange of the beam.

The clamping force does not result in a lack-of-fit strain or curvature across the adhesive joint, hence $\Delta \varepsilon_{p b}=\Delta \psi_{p b}=0$. The shear force at the end of the plate is equal to the clamping force,

$\left.V_{p}\right|_{x=0}=-10 \mathrm{kN}$ 
As shown in figure 6 , the clamping force results in only peel stress in the adhesive. The maximum compressive peel stress is $6.9 \mathrm{MPa}$, hence a clamping force of $19 \mathrm{kN}$ would be sufficient to react all of the peel stress associated with the prestress load case. A clamping force will not reduce the adhesive shear stress, although the shear strength of the adhesive is likely to be improved.

A prising load (with a load at the end of the plate applied away from the beam) could be used to assess the peel strength of an adhesive system.

\subsection{Discussion of bond stresses in simple load cases}

The load cases considered above investigate the adhesive stresses in a CFRP-strengthened cast iron beam. The relative importance of these load cases is noteworthy. In particular, the adhesive stresses due to temperature change are three times greater than those due to the live load carried by the beam. It is thus extremely important to consider temperature effects in metallic structures strengthened using carbon fibre composites and the designer must take care deciding the temperature range to which the beam will be subjected.

Prestressed strengthening results in high permanent adhesive stresses, and will usually require a permanent mechanical anchorage. The benefit of reacting the peel stresses using a clamping force at the end of the plate is shown in load case 5 .

The above examples illustrate how the bond analysis can be used to investigate the adhesive stresses due to a variety of simple load cases. The same analysis can be used to investigate any loading effect that can be expressed as a lack-of-fit across the adhesive joint. For example, the method can be used to investigate temperature gradients through the depth of a strengthened beam (rather than the uniform temperature case considered above).

\section{ADHESIVE STRESSES IN CASES OF MORE COMPLEX GEOMETRY}

The closed-form solution used in the above examples and given in Appendix A is only valid for:

- a continuous adhesive bond between the beam and plate;

- a sufficiently long adhesive layer, with no interaction between the two ends of the plate;

- no change in cross-section along the beam (ie: no change in plate, beam or adhesive dimensions). 
It is often desirable to analyse bonded plates with more complex geometry; for example, variations in plate thickness, beam depth, or adhesive stiffness. A numerical solution method, such as a finite-difference or finite element approach, is necessary where properties vary along the strengthened beam.

Appendix B outlines a finite-difference method for solving the governing equations derived in Appendix A. The approach is similar to that described in more detail by Deng, Lee and Moy [10]. Both use a constant node spacing, and require around 4000 nodes for convergence of the solution [10]. A more efficient solution (requiring around 500 nodes) can be achieved using variable node spacing, biased towards the position of the stress concentration. Further details of the variable node spacing solution (used to obtain the results given below) are given by the first author [13].

The following sections describe how the finite-difference solution can be used to investigate the use of stepped or tapered plates on adhesive stresses, and the sensitivity of a adhesive joint to defects. These examples are based on load case 1 (applied load) described above. Similar results would be obtained for the other load cases.

\subsection{Reduced plate thickness}

Reducing the plate thickness near its end reduces the maximum adhesive stresses; in particular, the peel stress is greatly reduced. Tests have confirmed that reducing the thickness of the end of the plate thickness improves the strength of the adhesive joint. The plate thickness can be reduced in two ways:

- $\quad$ stepped plates;

- gradually tapering the thickness of the plate.

\section{Stepped plates}

Where multiple layers of plates are used to make up the required area of strengthening, the ends of the plates can be stepped (as shown at the right end of figure 1). The finite-difference solution can be used to determine the adhesive stresses between the plates and the beam, and between the layers of plates. The stresses between the plates are found by treating the cast iron and upper plate as a composite section, with the lower plate as the strengthening. 
Figure 7 shows the beam-plate and plate-plate adhesive stresses for stepped plates subjected to the applied load considered in load case 1 . The plate thickness is $t_{p}=5.5 \mathrm{~mm}$ for $0<x<100 \mathrm{~mm}$, and $t_{p}=11 \mathrm{~mm}$ for $x>100 \mathrm{~mm}$. Stepping the plates gives a reduction in maximum shear stress of $30 \%$, and the maximum peel stress is reduced by $20 \%$ (compared to the unstepped case).

\section{Tapered plate}

Figure 8 shows the adhesive stresses in a tapered plate, with thickness increasing from $t_{p}=1 \mathrm{~mm}$ at $x=0$ to $t_{p}=11 \mathrm{~mm}$ at $x=100 \mathrm{~mm}$. The taper reduces the maximum shear stress by $25 \%$ and the maximum peel stress by $50 \%$ (compared to the unstepped case).

Further examples of the adhesive stresses with tapered plates are given by Deng, Lee \& Moy [10]. Frost, Lee \& Thompson [4] used Bessel functions to extend the analysis presented in Appendix A to cover tapers. However, the Bessel function solution requires zero thickness at the end of the plate, which is unlikely in practice.

\subsection{Defect sensitivity}

The finite-difference bond solution can also be used to assess the sensitivity of an adhesive joint to defects. Potential defects include air bubbles in the adhesive, surface contamination (such as grease), and corrosion products in the substrate (further information on bond defects can be found in reference [1]). All of these defects can be modelled by an absence of adhesive bond, so that $\tau_{a}=\sigma_{a}=0$ along the defect. This approach assumes that the defects are across the whole breadth, $b_{a}$, of the adhesive joint, allowing the worst case to be investigated.

\section{Surface preparation}

A common defect is poor surface preparation. The dashed line in figure 8 shows the adhesive stresses for the tapered plate, with a 50mm long surface preparation defect, starting $10 \mathrm{~mm}$ from the plate's end. The reduction in maximum shear stress achieved by using a tapered plate is lost; however, the maximum peel stress remains significantly lower than the untapered case. 


\section{End defect}

The ends of the plate are often the most difficult to bond; they are the most susceptible to environmental attack; and they correspond to the positions of maximum adhesive stress. Consequently, it is important to consider the effect of a bond defect propagating into the end of the adhesive joint.

Figure 9 shows the variation in maximum adhesive stresses with the length of an end defect for the tapered plate considered in figure 8 . The end defect significantly increases the maximum adhesive stresses, and could lead to unstable debonding of the strengthening plate from the beam.

\section{LIMITING ADHESIVE STRESSES}

An elastic bond stress analysis allows the maximum shear and peel stresses in an adhesive joint to be predicted. However, these are of little use unless appropriate limiting adhesive stresses can be determined for the joint being designed.

The supplier of the adhesive will typically report the strength of the adhesive obtained using a lap-shear test (such as BS5350 [14]). These results are the average shear stress along the specimen at failure, which are far lower than the peak stress reached in the lap-shear test. The peak shear and peel stress in a lap-shear specimen can be obtained by back-analysis, using a consistent linear-elastic analysis. C595 [1] describes this approach in detail, based on Goland \& Reissner's [15] analysis of a lap-shear test specimen. This is a low-order bond analysis, consistent with the bond analysis used for the strengthened beam.

It should also be recognised that the adhesive supplier's tests are undertaken on small coupons made under factory conditions. Designers must either ensure that the same standard of surface preparation and installation is obtained in the real adhesive joint, or use a reduced limiting stress, possibly obtained from lap-shear tests with a lower standard of surface preparation.

\section{CONCLUSIONS}

This paper has presented an elastic bond stress analysis suitable for design. The analysis is a powerful tool for investigating the strength of an adhesive joint between a strengthening plate and a beam. A number of illustrative examples have been explored, relating to a cast iron beam strengthened with a carbon fibre plate. These show how the adhesive stresses resulting from beam loading, temperature change and release of plate prestress can be calculated. In all cases, significant shear and 
peel stresses arise at the end of a strengthening plate, and these must be compared to the peak stresses obtained from a lap-shear test at failure. The high adhesive stresses due to temperature change are particular significant, as these are larger than the stresses due to applied load and will often govern design.

A variety of methods can be used to reduce the stresses acting across the adhesive joint. The end of the plate should be located in a region of low moment; a mechanical clamp can be used to react tensile peel stress; and the thickness of the plate can be reduced near to its end. A closed form solution allows strengthened beams with prismatic cross sections to be analysed and a finite-difference solution can be used to investigate more complex geometries, such as tapered or stepped plates.

It is also important to assess the sensitivity of the adhesive joint to bond defects such as poor surface preparation or environmental attack, which can significantly reduce the strength of the adhesive joint.

\section{ACKNOWLEDGEMENTS}

The closed-form bond analysis in Appendix A was initially developed by the authors while working for FaberMaunsell Ltd., and was included in CIRIA report C595 [1] 'Strengthening metallic structures using externally-bonded FRP'. The useful comments received from the C595 Steering Group members on the bond analysis are gratefully acknowledged, with particular thanks to Steve Denton of Parsons Brinkerhoff, and Simon Frost of AEA Technology.

\section{APPENDIX A - ELASTIC ANALYSIS OF AN ADHESIVE JOINT}

The geometry and material properties of the strengthened beam are shown in figure 2 and described in the main text.

\section{Methodology and assumptions}

The analysis of the strengthened beam section determines the distributions of shear stress $\left(\tau_{a}\right)$ and peel stress $\left(\sigma_{a}\right)$ acting across the adhesive joint, by examining compatibility requirements across the adhesive interface in the longitudinal direction (shear) and the through thickness direction (peel). 
The beam and/or plate will typically be under load at that the time the adhesive joint is formed, and it is important to consider the effect of these pre-strains. The compatibility conditions are thus written in terms of the lack-of-fit strains due to changes in the external loading after the adhesive set.

\section{Lack-of-fit across the adhesive joint}

The loading applied after the adhesive set is described by the change in stress resultants $\Delta N_{b}$, $\Delta M_{b}$ in the beam and $\Delta N_{p}, \Delta M_{p}$ in the plate. (As described in the main text, these are the stress resultants in the beam and plate if the adhesive joint is not present). The change in temperatures in the beam and plate after cure of the adhesive are $\Delta T_{b} \& \Delta T_{p}$.

Denoting the conditions at the time that the adhesive set as ' 0 ', and the conditions at the current time as ' $t$ ':

$$
\begin{array}{lll}
\Delta N_{b}=N_{b t}-N_{b 0} & \Delta M_{b}=M_{b t}-M_{b 0} & \Delta T_{b}=T_{b t}-T_{b 0} \\
\Delta N_{p}=N_{p t}-N_{p 0} & \Delta M_{p}=M_{p t}-M_{p 0} & \Delta T_{p}=T_{p t}-T_{p 0}
\end{array}
$$

These changes in the stress resultants result in a lack-of-fit in the strain across the adhesive interface. The strain $(\Delta \varepsilon)$ and curvature $(\Delta \psi)$ at the interface between the beam and the adhesive are:

$$
\Delta \varepsilon_{b a}=\frac{\Delta N_{b}}{E_{b} A_{b}}-\frac{\Delta M_{b} y_{b}}{E_{b} I_{b}}+\alpha_{b} \Delta T_{b} \quad \Delta \psi_{b}=\frac{\Delta M_{b}}{E_{b} I_{b}}
$$

Similarly, the strain and curvature at the plate-adhesive interface are:

$$
\Delta \varepsilon_{p a}=\frac{\Delta N_{p}}{E_{p} A_{p}}+\frac{\Delta M_{p} y_{p}}{E_{p} I_{p}}+\alpha_{p} \Delta T_{p} \quad \Delta \psi_{p}=\frac{\Delta M_{p}}{E_{p} I_{p}}
$$

The lack-of-fit strain across the adhesive joint is:

$$
\Delta \varepsilon_{p b}=\Delta \varepsilon_{p a}-\Delta \varepsilon_{b a}
$$

The lack-of-fit curvature across the adhesive joint is:

$$
\Delta \psi_{p b}=\Delta \psi_{p}-\Delta \psi_{b}
$$




\section{Equilibrium and constitutive relationships in the beam and plate}

The conditions in the plate and beam, connected by the adhesive joint, are described by the stress resultants $N_{p}, M_{p}, V_{p}$ in the plate (acting about the plate's centroid) and $N_{b}, M_{b}$ in the beam. As shown in figure 2 , these must be in equilibrium:

$$
N_{b}=-N_{p} \quad M_{b}=N_{p} z-M_{p}
$$

where $z$ is the lever arm between the centroids of the plate and the beam:

$$
z=y_{p}+t_{a}+y_{b}
$$

Constitutive relationships allow the strains $(\varepsilon)$ and curvatures $(\psi)$ in the beam and the plate to be expressed in terms of the plate stress resultants. In the plate:

$$
\varepsilon_{p}=\frac{N_{p}}{E_{p} A_{p}} \quad \psi_{p}=\frac{M_{p}}{E_{p} I_{p}}
$$

In the beam (using eqn. A7):

$$
\varepsilon_{b}=\frac{N_{b}}{E_{b} A_{b}}=-\frac{N_{p}}{E_{b} A_{b}} \quad \psi_{b}=\frac{M_{b}}{E_{b} I_{b}}=\frac{N_{p} z-M_{p}}{E_{b} I_{b}}
$$

\section{Equilibrium and constitutive relationships across the adhesive joint}

The mean shear stress $\left(\tau_{a}\right)$ and peel stress $\left(\sigma_{a}\right)$ acting across the adhesive joint are assumed to act about the mid-plane of the adhesive layer. By considering equilibrium of a short length of the plate (figure 2):

- Equilibrium in the $x$ direction gives the shear stress:

$$
\tau_{a}=\frac{1}{b_{a}} \frac{d N_{p}}{d x}
$$

- For rotational equilibrium (about the top-centre of the plate element):

$$
V_{p}=-\frac{d M_{p}}{d x}+y_{p} \frac{d N_{p}}{d x}=-\frac{d M_{p}^{*}}{d x}
$$

where $M_{p}{ }^{*}$ is the transformed moment about the plate-adhesive interface,

$$
M_{p}^{*}=M_{p}-y_{p} N_{p}
$$


- Equilibrium in the $y$ direction gives the peel stress:

$$
\sigma_{a}=\frac{1}{b_{a}} \frac{d V_{p}}{d x}=-\frac{1}{b_{a}} \frac{d^{2} M_{p}^{*}}{d x^{2}}
$$

The constitutive response of the adhesive is described by:

$$
\gamma_{a}=\frac{\tau_{a}}{G_{a}}=\frac{1}{b_{a} G_{a}} \frac{d N_{p}}{d x} \quad \varepsilon_{a}=\frac{\sigma_{a}}{E_{a}}
$$

\section{Shear compatibility of adhesive layer}

The shear stress, $\tau_{a}$, across the adhesive interface is determined by examining shear compatibility across the adhesive joint. The longitudinal displacements at the beam-adhesive $\left(u_{b a}\right)$ and plate-adhesive $\left(u_{p a}\right)$ interfaces can be expressed in terms of the horizontal and vertical displacements at the centroids of the beam $\left(u_{b}, v_{b}\right)$ and the plate $\left(u_{p}, v_{p}\right)$ :

$$
u_{p a}=\left(u_{p}+\frac{d v_{p}}{d x} y_{p}\right)+\Delta u_{p a} \quad u_{b a}=\left(u_{b}-\frac{d v_{b}}{d x} y_{b}\right)+\Delta u_{b a}
$$

where $u$ and $v$ are defined parallel to and in the same direction as $x$ and $y . \Delta u_{b a}$ and $\Delta u_{p a}$ are the lack-of-fit displacements associated with the change in load after the adhesive set (eqns. A1 \& A2).

Differentiating these equations with respect to $x$ gives the corresponding strains at the adhesive interfaces. Writing $\psi=d^{2} v / d x^{2}$ for the curvatures gives:

$$
\varepsilon_{p a}=\left(\varepsilon_{p}+\psi_{p} y_{p}\right)+\Delta \varepsilon_{p a} \quad \varepsilon_{b a}=\left(\varepsilon_{b}-\psi_{b} y_{b}\right)+\Delta \varepsilon_{b a}
$$

Assuming that the displacement field varies linearly through the adhesive layer, the average shear strain in the adhesive layer is given by:

$$
\gamma_{a}=\frac{\partial u}{\partial y}+\frac{\partial v}{\partial x}=\frac{u_{p a}-u_{b a}}{t_{a}}+\frac{1}{2}\left(\frac{\mathrm{d} v_{p}}{\mathrm{~d} x}+\frac{\mathrm{d} v_{b}}{\mathrm{~d} x}\right)
$$

Differentiating with respect to $x$ and substituting in the longitudinal strains gives:

$$
t_{a} \frac{d \gamma_{a}}{d x}=\varepsilon_{p}-\varepsilon_{b}+\psi_{p}\left(y_{p}+\frac{t_{a}}{2}\right)+\psi_{b}\left(y_{b}+\frac{t_{a}}{2}\right)+\Delta \varepsilon_{p b}
$$

Substituting for the strains using the constitutive relationships (eqns. A9, A10 \& A15) gives the compatibility equation for shear strain across the adhesive interface: 


$$
\begin{aligned}
\frac{t_{a}}{G_{a} b_{a}} \frac{d^{2} N_{p}}{d x^{2}=} & \left(\frac{1}{E_{p} A_{p}}+\frac{1}{E_{b} A_{b}}\right) N_{p}+\frac{M_{p}}{E_{p} I_{p}}\left(y_{p}+\frac{t_{a}}{2}\right) \\
& +\frac{N_{p} z-M_{p}}{E_{b} I_{b}}\left(y_{b}+\frac{t_{a}}{2}\right)+\Delta \varepsilon_{p b}
\end{aligned}
$$

The unknown variables, $N_{p} \& M_{p}$ are coupled. To simplify the solution, the difference between the beam and plate curvatures is assumed negligible for the purposes of determining $N_{p}$ :

$$
\psi_{p}=\frac{M_{p}}{E_{p} I_{p}}=\frac{N_{p} z-M_{p}}{E_{b} I_{b}}=\psi_{b}, \quad \text { hence, } \psi_{p}=\psi_{b}=\frac{N_{p} z}{E_{p} I_{p}+E_{b} I_{b}}
$$

$M_{p}$ can now be eliminated from eqn. A20, giving the governing equation for $N_{p}$ :

$$
-f_{1} \frac{d^{2} N_{p}}{d x^{2}}+f_{2} N_{p}=-\Delta \varepsilon_{p b}
$$

where:

$$
f_{1}=\frac{t_{a}}{G_{a} b_{a}} \quad f_{2}=\frac{1}{E_{p} A_{p}}+\frac{1}{E_{b} A_{b}}+\frac{z^{2}}{E_{p} I_{p}+E_{b} I_{b}}
$$

The solution of the governing equation is:

$$
N_{p}=N_{P S}+C_{1} e^{-\lambda x}+C_{2} e^{+\lambda x}
$$

$\lambda$ is a measure of the relative flexibility of the beam, plate and adhesive:

$$
\lambda=\sqrt{f_{2} / f_{1}}
$$

The particular solution, $N_{P S}$, is found by substitution of eqn. A24 into eqn. A22. Assuming that the lack-of-fit strain varies quadratically along the beam:

$$
\Delta \varepsilon_{p b}(x)=\Delta \varepsilon_{0}+\Delta \varepsilon_{1} x+\Delta \varepsilon_{2} x^{2}
$$

the particular solution is given by:

$$
N_{P S}=-\frac{1}{f_{2}}\left(2 \Delta \varepsilon_{2} \frac{f_{1}}{f_{2}}+\Delta \varepsilon_{p b}(x)\right)
$$

The constants of integration $\left(C_{1}, C_{2}\right)$ are found by applying suitable boundary conditions:

- the force in the plate is known at the discontinuity, 
- the force in the plate at another known point (for example the other end of the plate), $o r$, if the beam is symmetric the shear stress at the centre of the beam must be zero, hence (from eqn. A11), $\mathrm{d} N_{P} / \mathrm{d} x=0$.

For design purposes, it is usually be sufficient to assume that $C_{2}=0$, which implies that the length over which the stress concentration acts is small compared to the length of the adhesive joint. Applying the first boundary condition gives $C_{1}$. The axial force in the plate at position $x=0$ is denoted $\left.N_{p}\right|_{x=0}$. From eqn. A24:

$$
C_{1}=\left.N_{p}\right|_{x=0}-\left.N_{P S}\right|_{x=0}
$$

The shear stress in the adhesive can now be found using eqn. A11:

$$
\tau_{a}=\frac{1}{b_{a}}\left(\frac{\mathrm{d} N_{P S}}{\mathrm{~d} x}-C_{1} \lambda e^{-\lambda x}\right)
$$

The maximum shear stress occurs at $x=0$ :

$$
\tau_{\max }=\frac{1}{b_{a}}\left(\left.\frac{d N_{P S}}{d x}\right|_{x=0}-C_{1} \lambda\right)=-\frac{1}{b_{a}}\left(\frac{\Delta \varepsilon_{1}}{f_{2}}+C_{1} \lambda\right)
$$

Substituting for $\left.\frac{d N_{P S}}{d x}\right|_{x=0}$ from eqn. A27, $C_{1}$ from eqn. A28, and rearranging gives:

$$
\tau_{\max }=-\frac{1}{b_{a}}\left\{\frac{1}{f_{2}}\left(\lambda \Delta \varepsilon_{0}+\Delta \varepsilon_{1}+\frac{2}{\lambda f_{2}} \Delta \varepsilon_{2}\right)+\left.\lambda N_{p}\right|_{x=0}\right\}
$$

(The $\Delta \varepsilon_{1}$ term corresponds to St. Venant theory for shear stress in beam carrying shear load that causes lack-of-fit).

\section{Through thickness compatibility of adhesive layer}

The peel stress, $\sigma_{a}$, is found by examining the through-thickness strain, $\varepsilon_{a}$, in the adhesive, the difference in vertical displacement across the adhesive joint:

$$
\varepsilon_{a}=\frac{\partial v}{\partial y}=\frac{v_{p}-v_{b}}{t_{a}}+\frac{\Delta v_{p}-\Delta v_{b}}{t_{a}}
$$

Re-writing in terms of stress and differentiating twice with respect to $x$ : 


$$
\frac{t_{a}}{E_{a}} \frac{d^{2} \sigma_{a}}{d x^{2}}=\psi_{p}-\psi_{b}+\Delta \psi_{p b}
$$

Substituting for the curvatures using eqns. A3, A4, A6, A9 \& A10 gives the compatibility equation for peel:

$$
\frac{t_{a}}{E_{a}} \frac{\mathrm{d}^{2} \sigma_{a}}{\mathrm{~d} x^{2}}=\frac{M_{p}}{E_{p} I_{p}}-\frac{N_{p} z-M_{p}}{E_{b} I_{b}}+\Delta \psi_{p b}
$$

Re-writing this in terms of the transformed moment $\left(M_{p}{ }^{*}\right)$, and substituting for the peel stress $\left(\sigma_{a}\right)$ using eqn. A14 gives:

$$
a_{1} \frac{d^{4} M_{p}^{*}}{d x^{4}}+a_{2} M_{p}^{*}=a_{3} N_{p}-\Delta \psi_{p b}
$$

where:

$$
a_{1}=\frac{t_{a}}{E_{a} b_{a}} \quad a_{2}=\frac{1}{E_{p} I_{p}}+\frac{1}{E_{b} I_{b}} \quad a_{3}=\frac{z-y_{p}}{E_{b} I_{b}}-\frac{y_{p}}{E_{p} I_{p}}
$$

The solution of this equation is:

$$
\begin{aligned}
& M_{p}^{*}=M_{P S}^{*}+\left(C_{3} e^{-\beta x}+C_{5} e^{+\beta x}\right) \cos \beta x+\left(C_{4} e^{-\beta x}+C_{6} e^{+\beta x}\right) \sin \beta x \\
& \text { where } \beta=\left(\frac{a_{2}}{4 a_{1}}\right)^{0.25}
\end{aligned}
$$

The particular solution moment is found by substituting $\Delta \psi_{p b}$ into eqns. A33 \& A35. Assuming that the lack-of-fit curvature can be written as a quadratic function:

$$
\Delta \psi_{p b}(x)=\Delta \psi_{0}+\Delta \psi_{1} x+\Delta \psi_{2} x^{2}
$$

the particular solution moment is:

$$
M_{P S}^{*}=-\frac{1}{a_{2}} \Delta \psi(x)+\frac{a_{3}}{a_{2}} N_{P S}(x)+\frac{a_{3} C_{1}}{a_{1} \lambda^{4}+a_{2}} e^{-\lambda x}
$$

The transformed moment $M_{p}{ }^{*}$ (and hence the peel stress) approach zero for large values of $x$, hence the constants of integration $C_{5}$ and $C_{6}$ will be zero. $C_{3}$ and $C_{4}$ are found from the plate boundary conditions at $x=0$, where the bending moment and shear force are known: 


$$
\left.M_{p}^{*}\right|_{x=0}=\left.M_{p}\right|_{x=0}-\left.\left.y_{p} N_{p}\right|_{x=0} \quad V_{p}\right|_{x=0}=-\left.\frac{\mathrm{d} M_{p}}{\mathrm{~d} x}\right|_{x=0}
$$

Applying the moment boundary condition to eqn. A37 gives $C_{3}$ :

$$
\begin{aligned}
& C_{3}=\left.M_{p}^{*}\right|_{x=0}-\left.M_{P S}{ }^{*}\right|_{x=0} \\
& C_{3}=\left\{\left.M_{p}\right|_{x=0}-\left.y_{p} N_{p}\right|_{x=0}\right\}+\left\{\frac{\Delta \psi_{0}}{a_{2}}+\frac{a_{3}}{a_{2} f_{2}}\left(2 \Delta \varepsilon_{2} \frac{f_{1}}{f_{2}}+\Delta \varepsilon_{0}\right)-\frac{a_{3} C_{1}}{a_{1} \lambda^{4}+a_{2}}\right\}
\end{aligned}
$$

Applying the shear boundary condition to the first differential of eqn. A37 gives $C_{4}$ :

$$
C_{4}=-\frac{1}{\beta}\left[\left.V_{p}\right|_{x=0}+\left.\frac{\mathrm{d} M_{P S}^{*}}{\mathrm{~d} x}\right|_{x=0}-\beta C_{3}\right]=-\frac{1}{\beta}\left[\left.V_{p}\right|_{x=0}-\frac{\Delta \psi_{1}}{a_{2}}-\frac{a_{3} \Delta \varepsilon_{1}}{a_{2} f_{2}}\right]+C_{3}
$$

The peel stress is found by substituting the differentials of eqns. A37 and A40 into eqn. A14:

$$
\sigma_{a}=\frac{1}{b_{a}}\left[\begin{array}{l}
\frac{2}{a_{2}}\left(\Delta \psi_{2}+\frac{a_{3} \Delta \varepsilon_{2}}{f_{2}}\right)-\frac{a_{3} C_{1} \lambda^{2}}{a_{1} \lambda^{4}+a_{2}} e^{-\lambda x} \\
-2 \beta^{2} e^{-\beta x}\left(C_{3} \sin \beta x-C_{4} \cos \beta x\right)
\end{array}\right]
$$

The maximum value of the peel stress occurs at $x=0$ :

$$
\sigma_{\max }=\frac{1}{b_{a}}\left[\frac{2}{a_{2}}\left(\Delta \psi_{2}+\frac{a_{3} \Delta \varepsilon_{2}}{f_{2}}\right)-\frac{a_{3} C_{1} \lambda^{2}}{a_{1} \lambda^{4}+a_{2}}+2 \beta^{2} C_{4}\right]
$$

\section{APPENDIX B - FINITE-DIFFERENCE SOLUTION FOR VARYING SECTION PROPERTIES}

The governing equations for shear (eqn. A22) and peel (eqn. A35) are valid for plated beams with geometric and material properties that vary along its length, however, the coefficients $\left(f_{1}, a_{1}\right.$ etc $)$ vary in $x$, and a closed form solution is not possible. A finite-difference method can be used to find the adhesive stresses for cases with varying section properties.

A finite-difference solution using constant node spacing, $h$, is outlined below. The nodes are numbered $i=1 \ldots n$ (from $x=0$ to $x=L / 2$, the centre of the beam). Virtual nodes $(-1,0, n+1, n+2)$ are used to allow derivatives to be defined at nodes 1 and $n$. Superscripts are used to define node numbers in the following equations. 


\section{Shear stress solution}

The governing equation for the plate force (eqn. A22) can be written at each node $(i=1 \ldots n)$ along the beam:

$$
-f_{1}^{i} \frac{N_{p}^{i-1}-2 N_{p}^{i}+N_{p}^{i+1}}{h^{2}}+f_{2}^{i} N_{p}^{i}=-\Delta \varepsilon_{p b}^{i}
$$

Boundary condition (1): $N_{p}=\left.N_{p}\right|_{x=0}$ at $x=0$ (c.f. eqn. A28):

$$
N_{p}^{1}=\left.N_{p}\right|_{x=0}
$$

Boundary condition (2): $\tau=d N_{p} / d x=0$ at the centre of a symmetric beam (see discussion following eqn. A27):

$$
\frac{N_{p}^{n+1}-N_{p}^{n-1}}{2 h}=0
$$

These $n+2$ simultaneous equations are solved explicitly to find the plate force at each of the $n+2$ nodes. An implicit solution method (for example, by varying the shear stress at node 1 so as to satisfy boundary condition (2) at node $n$ ) will not be successful, due to the sensitivity of conditions at node $n$ to small changes at node 1 .

The shear stress distribution follows from eqn. A11:

$$
\tau_{a}^{i}=\frac{1}{b_{a}^{i}} \frac{N_{p}^{i+1}-N_{p}^{i-1}}{2 h}
$$

\section{Peel stress solution}

A fourth-order finite-difference solution is required to find the transformed plate moment. The governing equation for peel (eqn. A35) is written at each node $(i=1 \ldots n)$ along the beam:

$$
a_{1}^{i} \frac{M_{p}^{*(i-2)}-4 M_{p}^{*(i-1)}+6 M_{p}^{*_{i}}-4 M_{p}^{*(i+1)}+M_{p}^{*(i+2)}}{h^{4}}+a_{2}^{i} M_{p}^{*_{i}}=a_{3} N_{p}^{i}-\Delta \psi_{p b}^{i}
$$

Boundary condition (1): $M_{p}^{*}=\left.M_{p}^{*}\right|_{x=0}$ at $x=0$ (c.f. eqn. A41):

$$
M_{p}^{* 1}=\left.M_{p}^{*}\right|_{x=0}
$$


Boundary condition (2): $V_{p}=d M_{p}^{*} / d x=\left.V_{p}\right|_{x=0}$ at $x=0$ (c.f. eqn. A41):

$$
\frac{M_{p}^{*(-2)}-8 M_{p}^{*(-1)}+8 M_{p}^{* 1}+M_{p}^{* 2}}{12 h}=\left.V_{p}\right|_{x=0}
$$

Boundary condition (3): $V_{p}=d M_{p}^{*} / d x=0$ at the centre of a symmetric beam:

$$
\frac{M_{p}^{*(n-2)}-8 M_{p}^{*(n-1)}+8 M_{p}^{*(n+1)}+M_{p}^{*(n+2)}}{12 h}=0
$$

Boundary condition (4): $d^{3} M_{p}^{*} / d x^{3}=0$ at the centre of a symmetric beam:

$$
\frac{-M_{p}^{*(n-2)}+2 M_{p}^{*(n-1)}-2 M_{p}^{*(n+1)}+M_{p}^{*(n+2)}}{2 h}=0
$$

These $n+4$ simultaneous equations are solved explicitly for the plate force at each of the $n+4$ nodes. The peel stress distribution follows from eqn. A14:

$$
\sigma_{a}^{i}=-\frac{1}{b_{a}^{i}}\left[\frac{-M_{p}^{*(i-2)}+16 M_{p}^{*(i-1)}-30 M_{p}^{* i}+16 M_{p}^{*(i+1)}-M_{p}^{*(i+2)}}{12 h^{2}}\right]
$$




\section{REFERENCES}

[1] Cadei JMC, Stratford TJ, Hollaway LC, Duckett WG. C595 - Strengthening metallic structures using externally-bonded fibre-reinforced-polymers. London: CIRIA, 2004.

[2] Buyukozturk O, Gunes O, Karaca E. Progress on understanding debonding problems in reinforced concrete and steel members strengthened using FRP composites. Construction and building materials 2003; 18: 9-19

[3] Denton SR. Analysis of stresses developed in FRP plated beams due to thermal effects. In: Teng J.G., editor. Proceedings of the international conference on FRP composites in civil engineering. Hong Kong: Elsevier, 2001. p. 527-536.

[4] Frost S, Lee RJ, Thompson VK. Structural integrity of beams strengthened with FRP plates Analysis of the adhesive layer. In: Force M.C., editor. Structural faults \& repair 2003. London, 2003.

[5] Miller TC, Chajes MJ, Mertz DR, Hastings JN. Strengthening of a steel bridge girder using CFRP plates. New York City bridge conference, 2001.

[6] Roberts TM. Approximate analysis of shear and normal stress concentrations in the adhesive layer of plated RC beams. The structural engineer 1989; 10(2):.229-233

[7] Albat AM, Romilly DP. A direct linear-elastic analysis of double symmetric bonded joints and reinforcements. Composite science and technology 1999; 59(7): 1127-1137.

[8] Smith ST, Teng JG. Interfacial stresses in plated beams. Engineering structures 2001; 23: 857871.

[9] Yang J, Teng JG, Chen JF. Interfacial stresses in soffit-plated reinforced concrete beams. Proc inst civil engineers: Structures and buildings 2004; 157(1): 77-89.

[10] Deng J, Lee MMK, Moy SSJ. Stress analysis of steel beams reinforced with a bonded CFRP plate. Composite structures 2004; 65: 205-215

[11] Moy SSJ, editor. FRP composites - Life Extension and Strengthening of Metallic Structures. London: Institution of Civil Engineers, 2001. p.33-35

[12] BD 37/01. Loads for Highway Bridges, London: The Highways Agency, 2001. 
[13] Stratford TJ. Further details of the finite-difference solution used can be found at http://www.see.ed.ac.uk/ tstratfo/adhesivejoints/, including downloadable Matlab files. A further paper is in preparation.

[14] BS 5350-C5:2002. Methods of test for adhesives. Determination of bond strength in longitudinal shear for rigid adherends. British Standards Institute 2002.

[15] Goland M, Reissner E. The stresses in cemented joints. Journal of applied mechanics, Transactions of ASME 1944; 11: A11-27. 


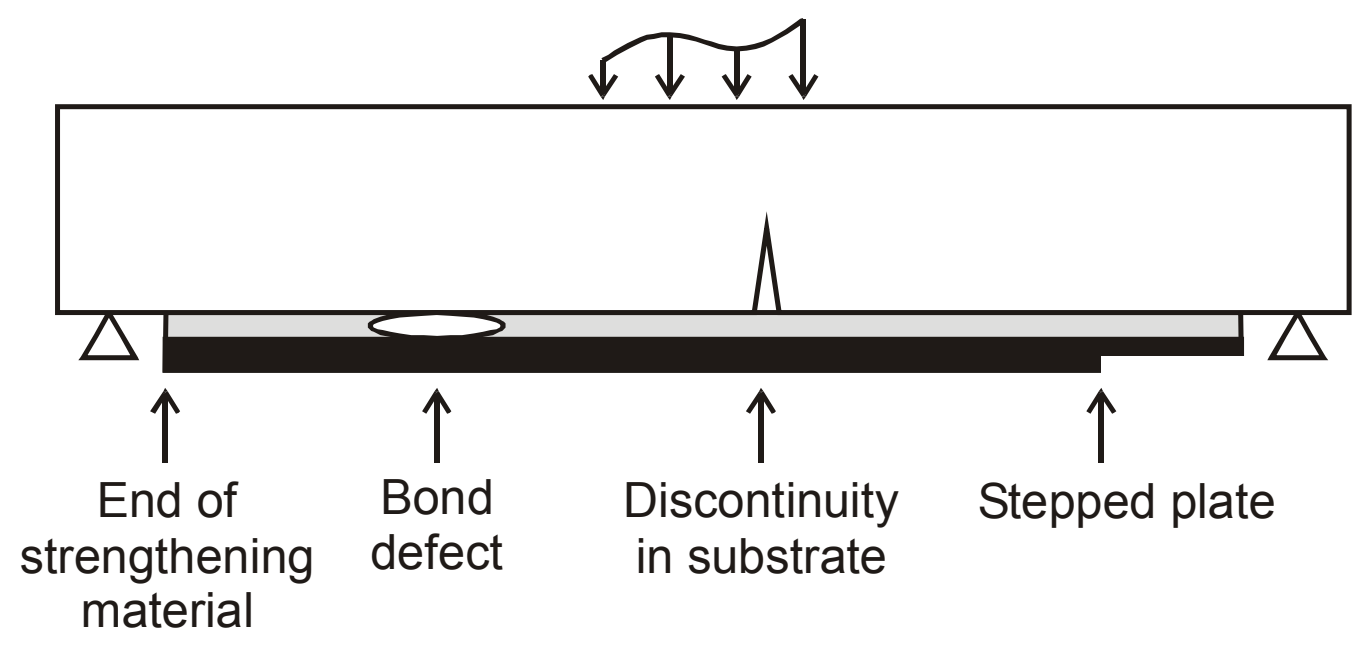

Figure 1 - Geometric discontinuities that cause adhesive stress concentrations. 


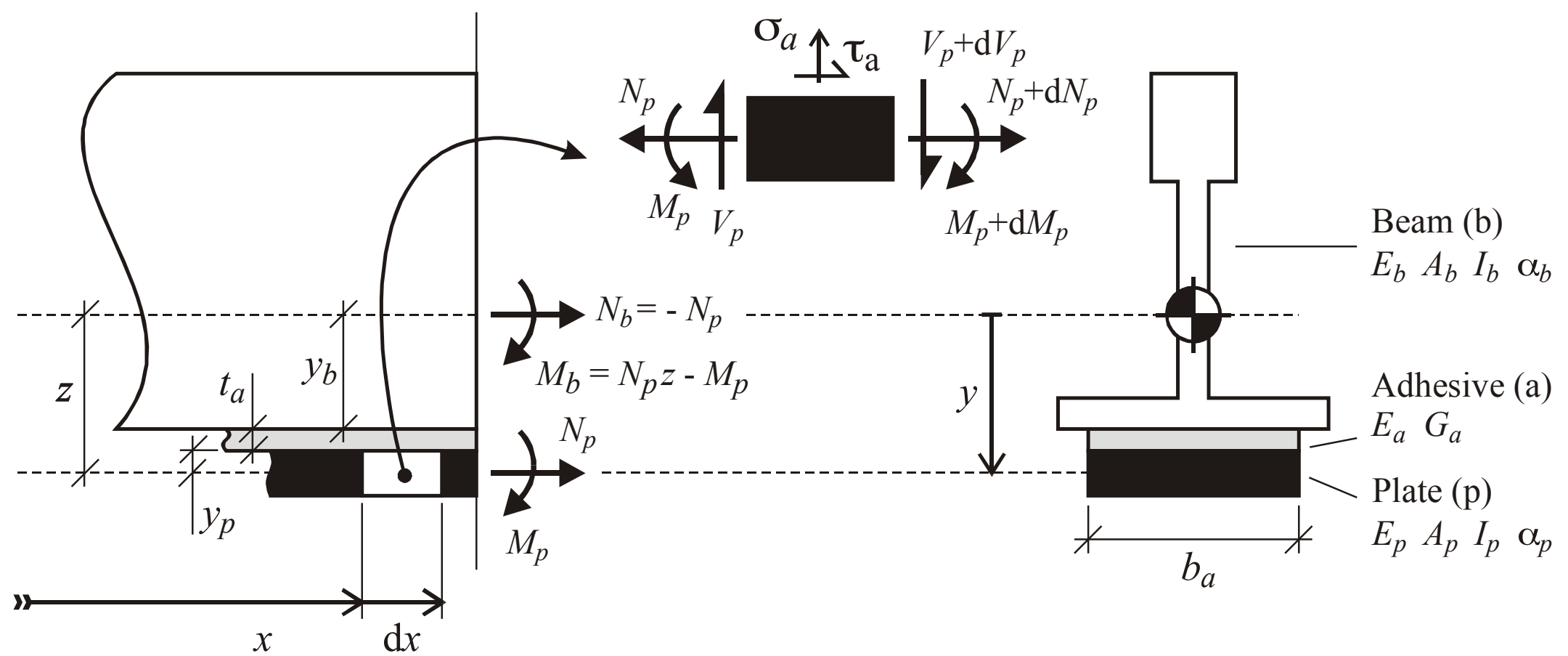

Figure 2 - Equilibrium between the plate, beam and adhesive joint, and the geometry and material properties of the bonded plate and beam. 

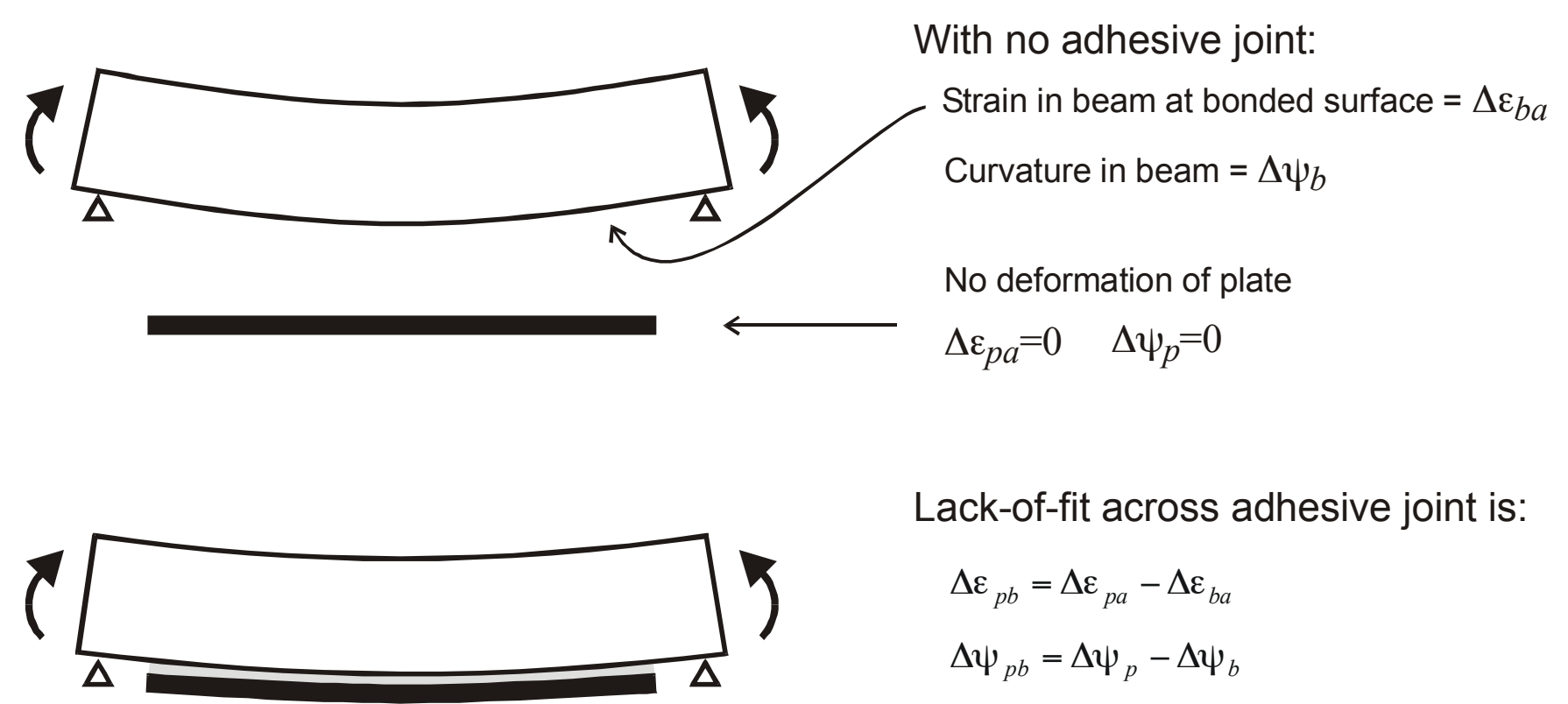

Figure 3 - Lack-of-fit for a strengthened beam carrying pure moment. 

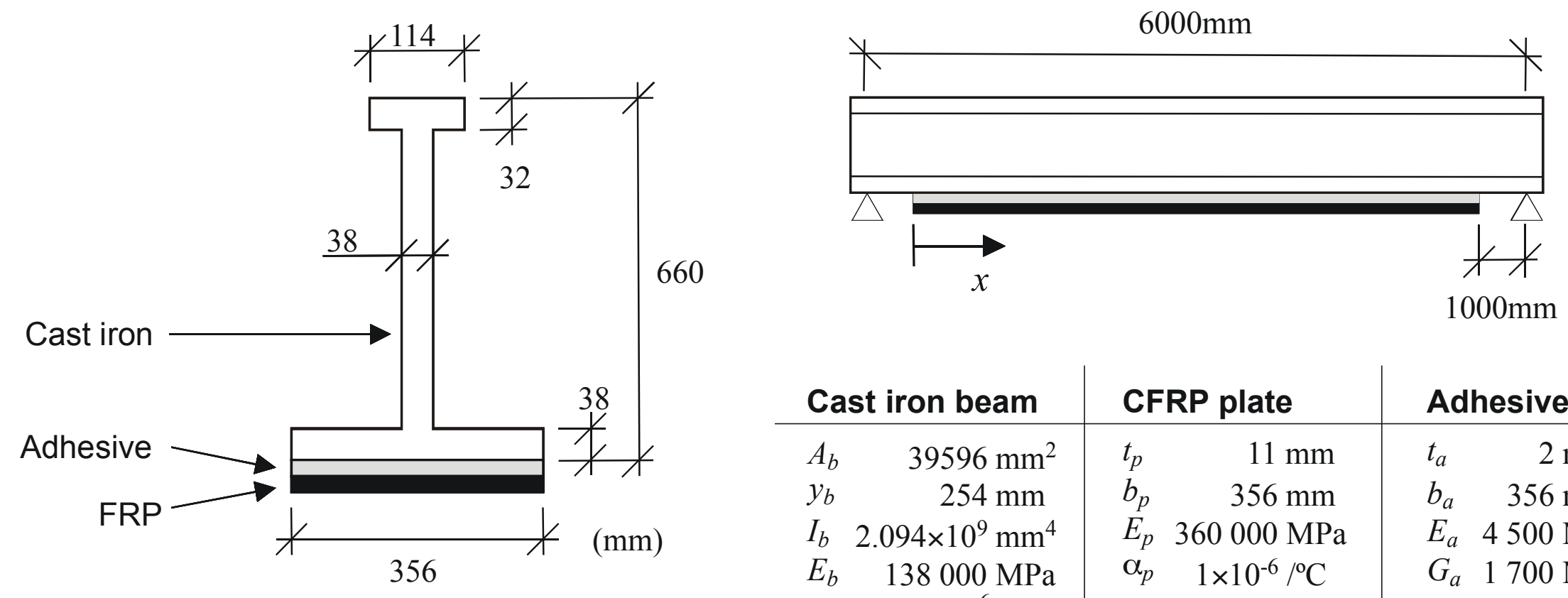

\begin{tabular}{|c|c|c|c|}
\hline \multicolumn{2}{|c|}{ Cast iron beam } & CFRP plate & Adhesive \\
\hline$A_{b}$ & $39596 \mathrm{~mm}^{2}$ & $11 \mathrm{~mm}$ & $2 \mathrm{~mm}$ \\
\hline$y_{b}$ & $254 \mathrm{~mm}$ & $356 \mathrm{~mm}$ & $356 \mathrm{~mm}$ \\
\hline$I_{b}$ & $2.094 \times 10^{9} \mathrm{~mm}^{4}$ & $E_{p} 360000 \mathrm{MPa}$ & $E_{a} \quad 4500 \mathrm{MPa}$ \\
\hline$E_{b}$ & $138000 \mathrm{MPa}$ & $\alpha_{p} \quad 1 \times 10^{-6} /{ }^{\circ} \mathrm{C}$ & $G_{a} 1700 \mathrm{MPa}$ \\
\hline$\alpha_{b}$ & $11 \times 10^{-6} /{ }^{\circ} \mathrm{C}$ & & \\
\hline
\end{tabular}

Figure 4 - Details of the strengthened cast iron considered in the examples. 

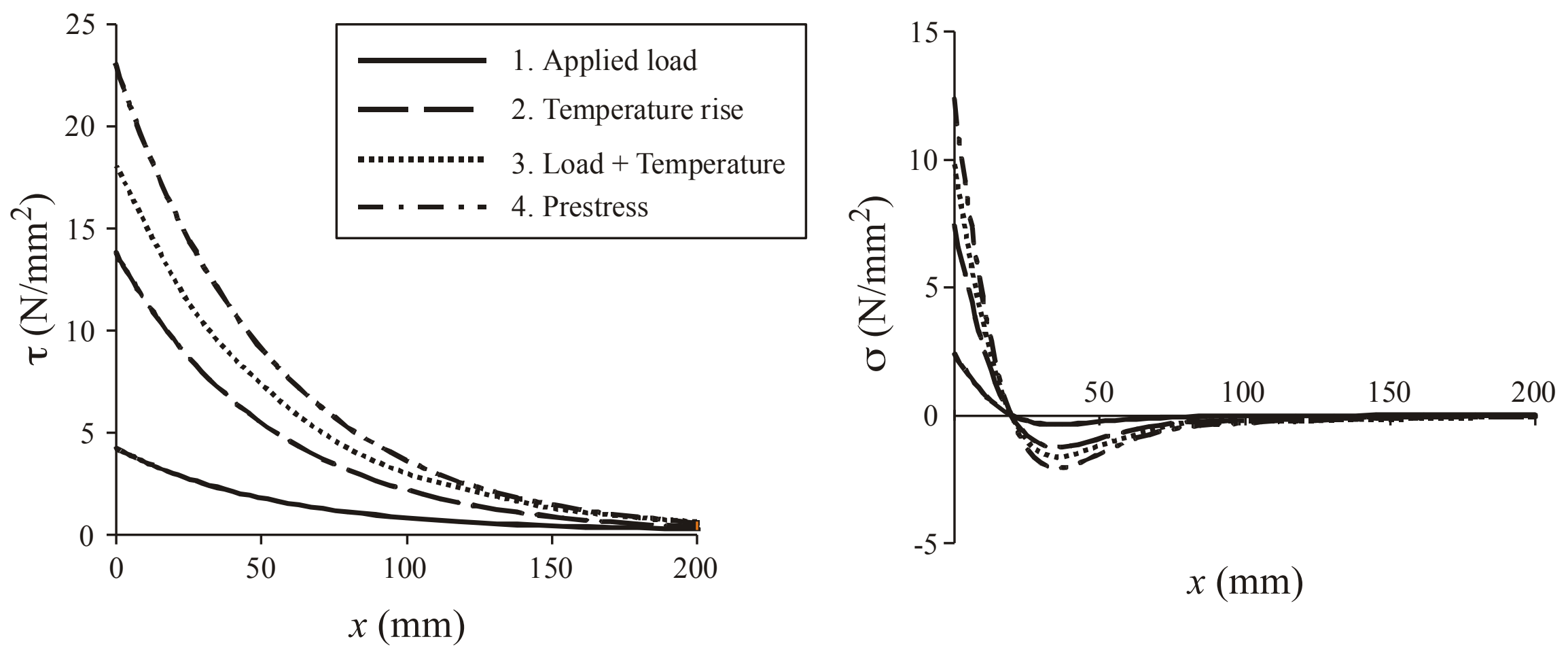

Figure 5 - Adhesive stresses due to (1) applied load, (2) a uniform temperature rise of $30^{\circ} \mathrm{C}$, and (3) release of prestressed plate. 


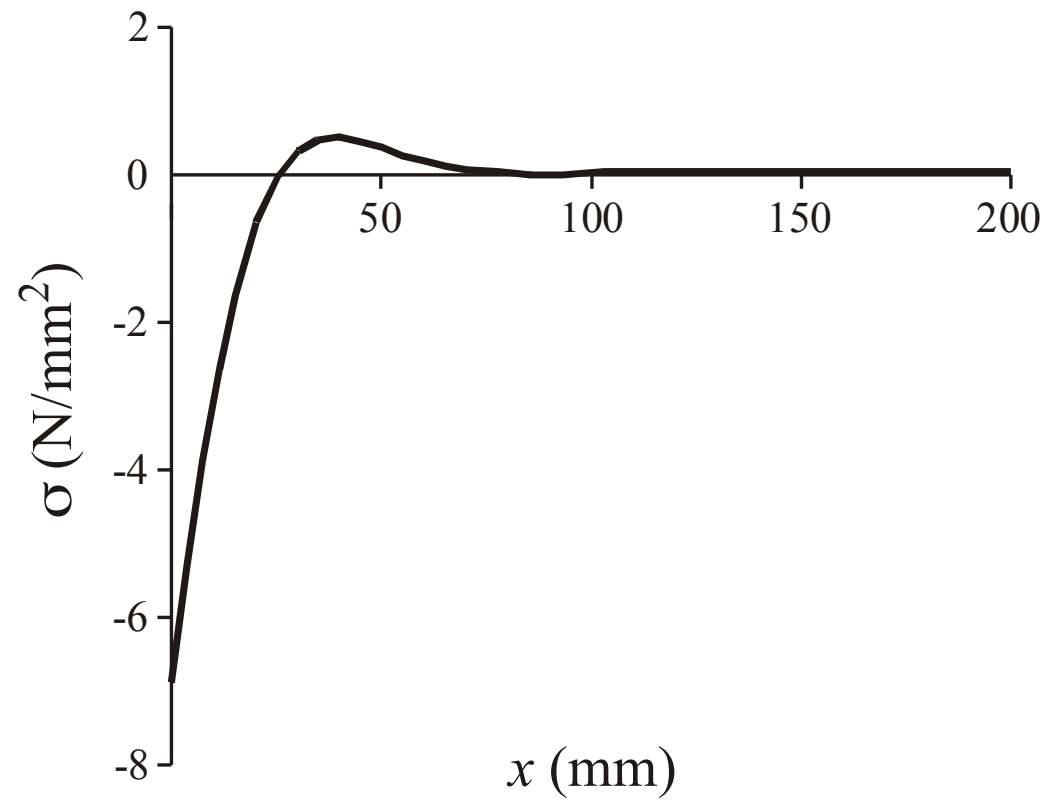

Figure 6 - Adhesive stresses due to a $10 \mathrm{kN}$ prising force applied at the end of the plate. 

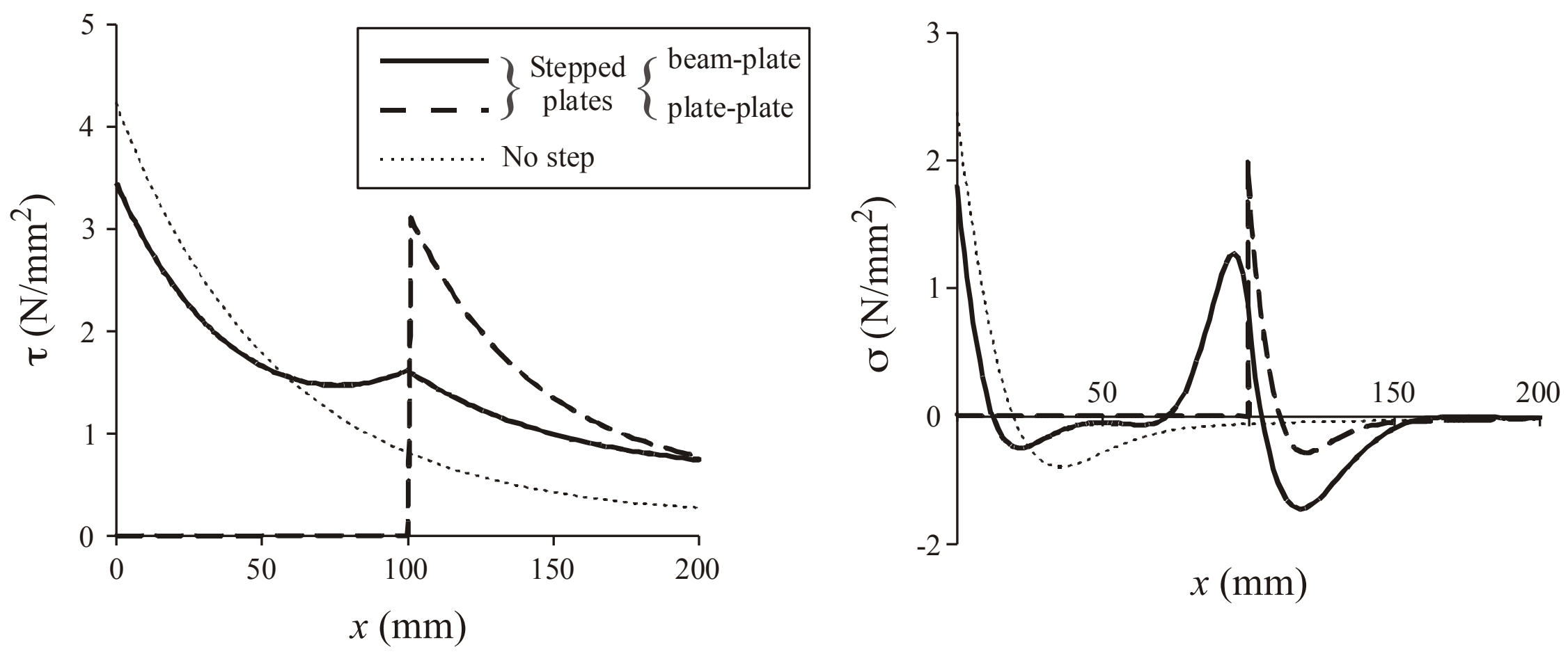

Figure 7 - Adhesive stresses in a beam strengthened with two stepped plates subjected to applied load, at (1) the beam-plate interface and (2) between the plates. 

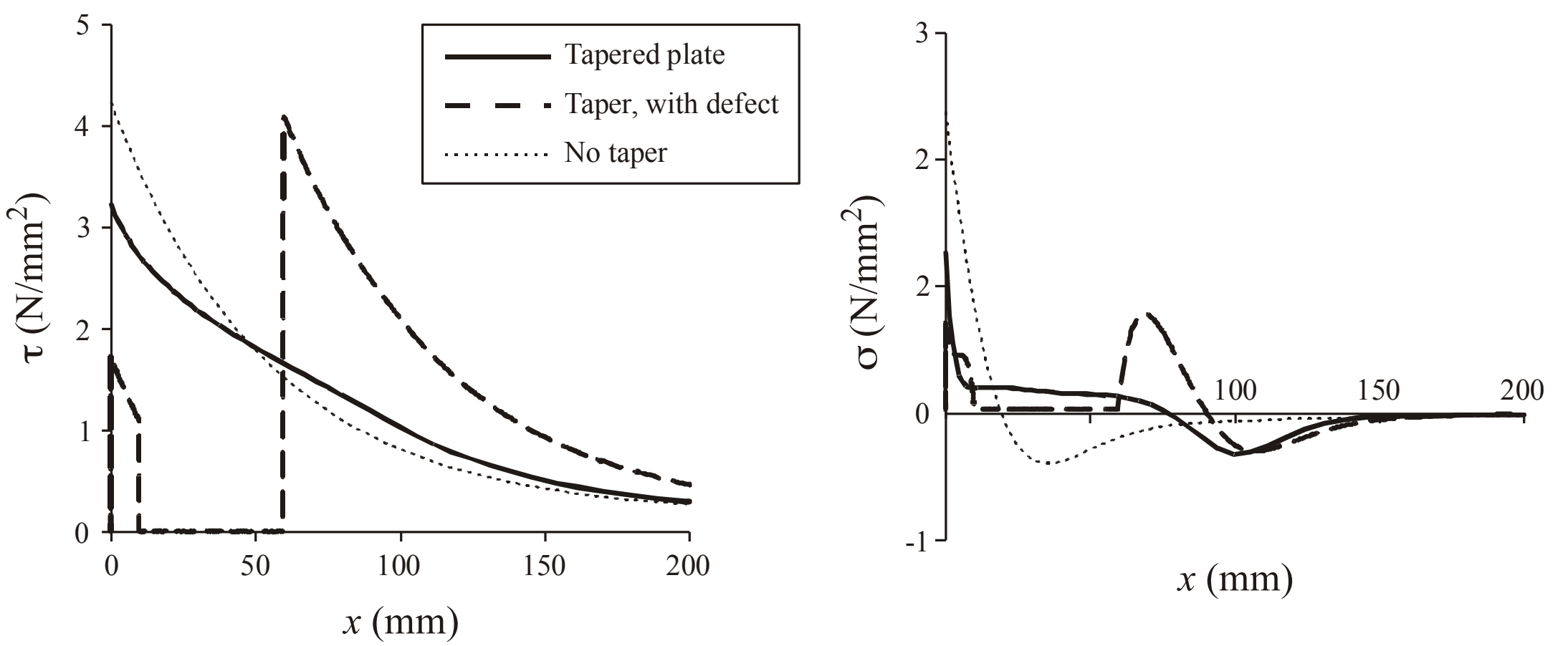

Figure 8 - Adhesive stresses in a beam strengthened with a tapered plate, subjected to applied load: (1) with a complete adhesive layer and (2) with a 50mm long defect in the adhesive layer. 


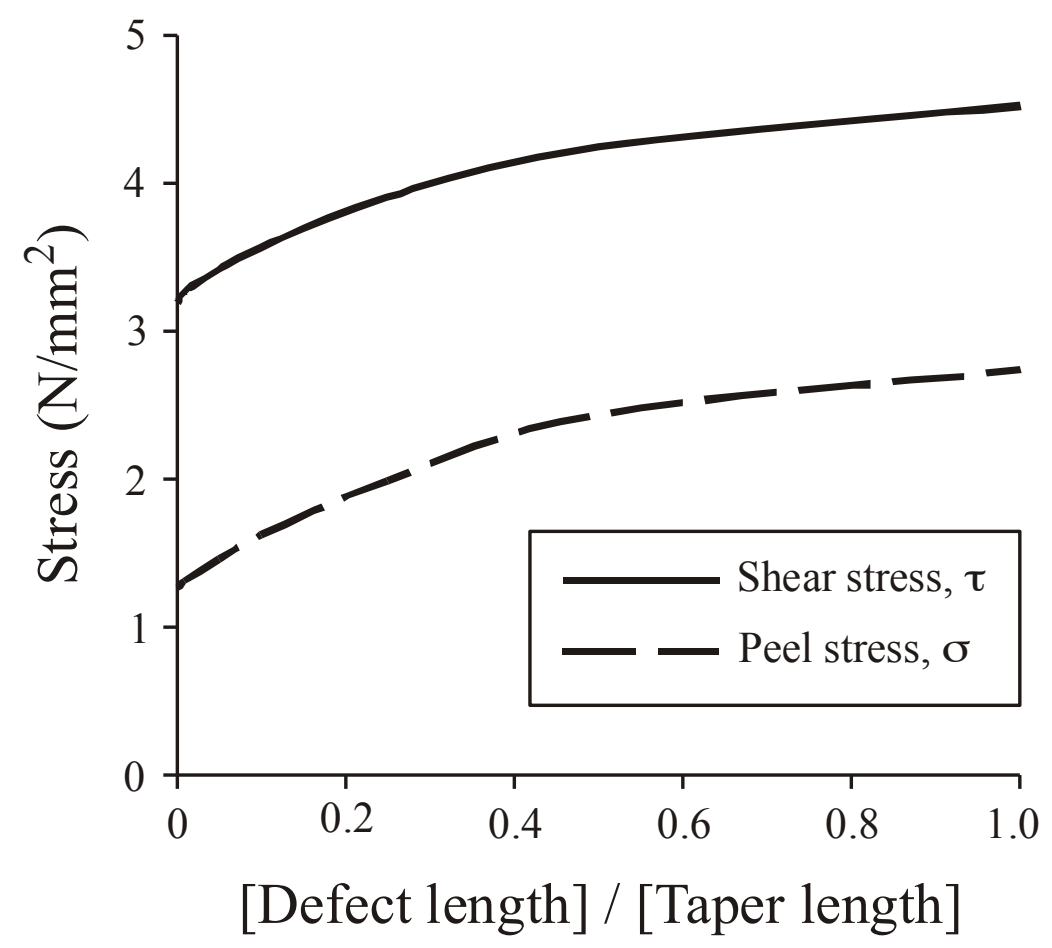

Figure 9 - The change in maximum adhesive stresses with a tapered plate due to propagation of a bond defect into the end of the adhesive joint. 